\title{
LES LOSS PREDICTION IN AN AXIAL COMPRESSOR CASCADE AT OFF-DESIGN INCIDENCES WITH FREE STREAM DISTURBANCES
}

\author{
John Leggett* \\ University of Southampton \\ Southampton, UK \\ Email:j.leggett@soton.ac.uk \\ Aamir Shabbir \\ GE Aviation \\ Cincinnati, $\mathrm{OH}, \mathrm{USA}$ \\ Richard Sandberg \\ University of Melbourne \\ Melbourne, Australia
}

\author{
Stephan Priebe \\ GE Global Research \\ Niskayuna, NY, USA
}

\author{
Vittorio Michelassi \\ General Electric Oil\&Gas \\ Florence, Italy
}

\author{
Edward Richardson \\ University of Southampton \\ Southampton, UK
}

\begin{abstract}
Axial compressors may be operated under off-design incidences due to variable operating conditions. Therefore, a successful design requires accurate performance and stability limits predictions under a wide operating range. Designers generally rely both on correlations and on RANS, the accuracy of the latter often being questioned. The present study investigates profile losses in an axial compressor linear cascade using both RANS and wall-resolved Large Eddy Simulation (LES), and compares with measurements. The analysis concentrates on "loss buckets", local separation bubbles and boundary layer transition with high levels of free stream turbulence, as encountered in real compressor environment without and with periodic incoming wakes. The work extends previous research with the intention of furthering our understanding of prediction tools and improving our quantification of the physical processes involved in loss generation. The results show that while RANS predicts overall profile losses with good accuracy, the relative importance of the different loss mechanisms does not match with LES, especially at offdesign conditions. This implies that a RANS based optimisation of a compressor profile under a wide incidence range may require a thorough LES verification at off-design incidence.
\end{abstract}

\section{INTRODUCTION}

Axial compressors, in both power generation and aeropropulsion, are asked to operate under a wide range of conditions with strong off-design incidences. The full exploitation of Reynolds Averaged Navier Stokes (RANS) in the design of axial compressors is limited by two key problems: 1 - At design conditions, small efficiency and deviation angle inaccuracies at single airfoil level can have strong snow-ball effect in multistage compressors compromising stage matching. 2 - Off-design conditions require the ability to accurately predict stall, a realm where RANS has well-known problems.

With mismatches in predicted and actual performance likely to result in costly redesigns of the compressor, the emphasis on accurate predictions at the design stage is becoming ever more important, especially with the current design trend with stages more highly loaded and the first stages transonic [1]. Therefore, improvements in CFD techniques are already a large research area, with work on improving current models and developing new techniques currently taking place. In many cases the computational cost of applying high fidelity methods, like Large Eddy Simulation (LES) to relevant flows is still prohibitive and options are often limited to RANS. The use of hybrid methods such as Detached Eddy Simulations present an attractive means of improving predictions with limited cost increase, but these methods are still exposed to many of the shortcomings of RANS.

It is well known that RANS accuracy can be at risk, even at design point, when periodic disturbances (like incoming wakes) and turbulence (that naturally builds up in a multistage compressor) occur at length scales similar to those RANS or URANS is modelling [2,3]. Accuracy can further 
deteriorate when large scale/low frequency structures coexist with background turbulence, a situation that can occur at off-design conditions where large separations and turbulent shedding can occur.

As it seems unlikely that a replacement for RANS modelling that holds valid in a wide enough operating range will be found in the near future it is important to understand its performance prediction characteristics. Hence, in an attempt to ensure that decisions made at the design stage are made with a proper understanding of the predictions results it is important to compare RANS not only with measurements, but also with higher accuracy methods to both quantify the differences and understand the physics.

Large Eddy Simulations can provide the required improved accuracy and serve as reference for comparison with standard methods. This is becoming more viable for engine Reynolds number flows, albeit limited to only a few runs. With access to these improved methods a better understanding of the current design methods can be gained and their limitations can be quantified. Such practice proved successful for Low-Pressure-Turbines, [4, 5], where RANS closure assumptions were benchmarked by using both DNS and LES flow fields. The purpose of this paper is to extended such practice to axial compressors cascades.

Axial compressors profiles require more attention to offdesign conditions as opposed to axial turbines. As turbine airfoils, up to high-subsonic conditions, show a moderate performance degradation at off-design, the existing DNS and LES investigations concentrated on design conditions and on combinations of design parameters that did not deviate from design [6]. On the contrary, the design of an axial compressor requires a much more detailed investigation of off-design operability, or stall margin, at both positive and negative incidences. In particular, even the post-stall behaviour of an airfoil is of interest as the wakes strength and frequency of the separation bubble burst are important information that generally become available only in the testing phase. Therefore, the analyses described in this paper consider both design and off-design conditions of a typical axial compressor vane in a linear cascade configuration. With the aid of a set of high-resolution LES, the performance of current design methods, specifically Reynolds Averaged Navier Stokes (RANS), is also reviewed to investigate the physics involved.

Extending the need for better prediction to time dependent phenomena such as wake interactions is a logical next step as these interactions are fundamental in today's compressor designs. The influence of discrete free stream disturbances on the performance of down stream blade rows can affect stage matching and overall performance. In compressors the combination of reduced frequency and flow coefficient keeps periodic incident wakes distinct, while in turbines wakes have been demonstrated to merge sometimes before reaching the downstream airfoils [4]. As a result, incoming wakes do have distinct effects on a compressor blade performance. It is shown that wakes cause large variations of the blade boundary layer, with changes in the transition mechanisms and transition point over the blade surface [7]. Capturing these variations is vital to properly predicting the performance of a compressor stage [8]. However it is often too costly to simulate wake interaction fully which has resulted in simplification techniques to improve simulations predictions without excessive expense.

This paper addresses these points, and, after a preliminary validation built upon a previous paper from the authors [9], it focuses on a detailed loss generation mechanism analysis to highlight the limitations and performance of the CFD tools used.

\section{CASE SET-UP}

The simulations focus on the performance prediction of a NACA 65 V103 airfoil, that is representative of a realistic axial compressor mid-section. The airfoil performance has been investigated by Leipold et al. [10] and Hilgenfeld \& Pfitzner [11] who concentrated on the midspan section of a linear cascade rig to minimize the complex flow interactions that end walls and tip leakages cause. The experiments cover design and off-design incidence as wall as the impact of incoming wakes. The set-up is outlined in Figure 1 with a schematic representation of the cascade. The upstream moving bars used for wake generation, placed at a distance of 0.38 chord upstream of the leading edge, are removed for off-design incidence conditions. The nominal simulation parameters are outlined in Table 1.

The simulations concentrate on the mid-span section at an axial chord Reynolds number of $\approx 300,000$ and an inlet Mach number of 0.67. For the cases looking at the effects of off-design incidence a nominal level of $3 \%$ free stream turbulence is added to ensure engine-like operating conditions. The incident wake case however was run without free stream turbulence, as for turbine flow calculations background turbulence had marginal effect when combined with incoming wakes [4]. The final running conditions for the two cases are given in Table 2. It is noted that the Mach number for the wake case is slightly lower than the desired 0.67 due to a larger than predicted slowdown of the flow due to the bar blockage.

The mesh is a structured H-grid with an embedded Ogrid type as outlined by the dashed lines in Figure 2. The inlet length upstream of the blade mesh was deliberately kept short to allow the addition of the moving bar mesh (see Figure 2 outlined in purple). For the off-design simulations the moving bar mesh is replaced with a uniform mesh. The bar mesh is also an $\mathrm{H}$-grid with embedded $\mathrm{O}$-grid structure repeated for each bar. The computational domain extends 0.5 chord upstream of the profile for the clean inlet cases and 0.6 chord for the moving bar case, while the downstream and span-wise extents were kept constant for all simulations at 1 chord and 0.2 chord lengths respectively. The blade mesh contains 603,136 points in the plane and 128 modes in the span giving a total of $\approx 155 \mathrm{M}$ points. For the appended upstream mesh, the off-design mesh contains 24,576 points in the plane and the moving bar mesh 132,096. Both have 128 modes in the span giving $\approx 6 \mathrm{M}$ and $\approx 34 \mathrm{M}$ points respectively.

The LES simulations are wall resolved. Therefore, the 
blade mesh has a wall resolution of $\Delta \mathrm{x}^{+} \approx 15-20, \Delta \mathrm{y}^{+} \approx$ 1.5 and $\Delta \mathrm{z}^{+} \approx 12-22$, with the lowest resolution found near the stagnation point of the leading edge. The resolution of the bar mesh was restricted to the smallest cell size of the blade to ensure no time step penalty was incurred, resulting in a resolution of $\Delta \mathrm{x}^{+} \approx 5-12, \Delta \mathrm{y}^{+} \approx 2-4$ and $\Delta \mathrm{z}^{+} \approx 15-27$ for the bars. The 2D RANS simulations are performed on a 151,000 points mesh with a wall resolution of $y^{+}<1$ for both suction and pressure surfaces.

The boundary conditions for the simulations are the same for all cases apart from the addition of free-stream turbulence in the clean inlet cases. The exit boundary condition is a non-reflective characteristic boundary prescribing pressure only, while the blade and bar walls are non-slip adiabatic. Finally the span-wise and pitch-wise boundaries are periodic.

For the cases requiring free-stream turbulence the synthetic inlet turbulent length scales are set to $10 \%$ chord in the pitch wise and stream wise directions and 5\% chord in the span wise direction, limited by the domain length and filter width. The length scale of the free-stream turbulence was not explicitly given in the experimental reference cases so the choice of length scale is based on expected turbulent length scales shed from upstream blades in a real engine.

The incident wake case replicates the set-up of Hilgenfeld \& Pfitzner [11] run with a reduced frequency of 0.66 , defined as $F_{\text {red }}=\frac{V_{b a r} C}{P_{b a r} U}$, flow coefficient of $\approx 5.7$, defined as $\frac{U}{V_{b a r}}$ at a Reynolds number of 300,000 and Mach number of 0.67 . While the bar speed gives a flow coefficient that is not representative of typical engine conditions, the case does provide a good starting point for comparison with experimental data for what concerns the capability of the code to describe the details of the incoming wakes. To investigate a case with realistic periodic incoming wakes we set-up an additional numerical case the characteristics of which are summarized in Table 2 under the Wake New column. The Wake New case uses the same set-up as the reference wake case but with a single bar placed up-stream with $t_{\text {bar }}=t$. The results of the Wake New case are discussed in the final section of the paper and these provide further insight into the effects wakes have on the blade performance.

The reference planes used throughout the study for normalization, inlet parameters and loss calculations are 0.3 chord upstream of the leading edge and 0.1 chord downstream of the trailing edge. The inlet reference plane lies between the moving bars and the blade.

Table 1. Nominal simulation parameters

\begin{tabular}{|c|c|}
\hline $\mathrm{Ma}=0.67$ & $\alpha=42^{\circ}$ \\
\hline $\mathrm{Re}=300,000$ & $\mathrm{t} / \mathrm{C}=0.59$ \\
\hline $\mathrm{d} / \mathrm{C}=0.059$ & $\beta_{s}=112.5^{\circ}$ \\
\hline$c_{\text {bar }} / C=0.0098$ & $t_{\text {bar }}=\mathrm{t} / 3$ \\
\hline
\end{tabular}

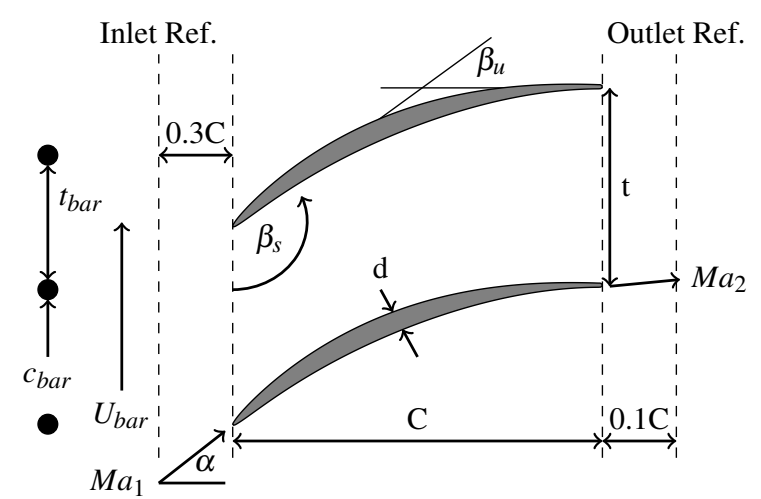

Fig. 1. Schematic of the NACA 65 linear compressor cascade used here, based on outline in Hilgenfeld \& Pfitzner [11]

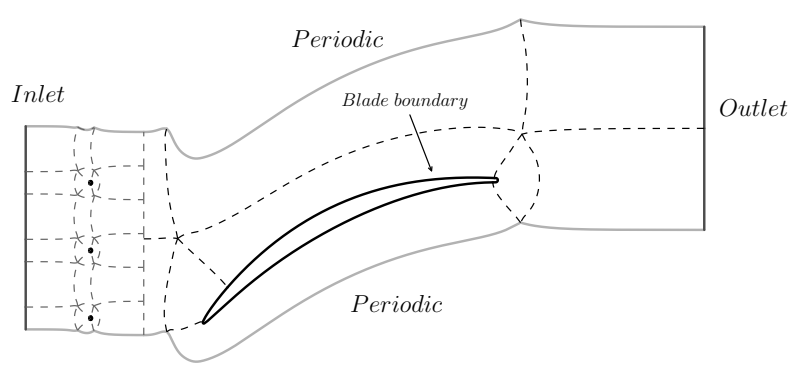

Fig. 2. Boundary conditions and multi block layout of the linear cascade. Moving bar mesh shown in purple

Table 2. Final simulation conditions

\begin{tabular}{|l|c|c|c|}
\hline \multicolumn{4}{|c|}{ Table 2. Final simulation conditions } \\
\hline Reynolds & Off-design & Wake [11] & Wake New \\
\hline Mach & $289 \mathrm{~K}$ & $280 \mathrm{~K}$ & $314 \mathrm{~K}$ \\
\hline Flow Angle & 49.2 & 0.63 & 0.658 \\
\hline $\begin{array}{l}\text { \% Tu at LE } \\
\text { (pitch average) }\end{array}$ & 3.5 & 43. & 43.9 \\
\hline Reduced Freq. & NA & 0.66 & 3.5 \\
\hline Flow coef. & NA & 5.7 & 0.89 \\
\hline
\end{tabular}

\section{NUMERICS AND COMPUTATIONAL PERFOR- MANCE}

The LES simulations are performed by the in house code HiPSTAR [12]. The code is a compressible Navier-Stokes multi-block structured solver, employing finite differences in the plane and a Fourier spectral method in the span-wise direction. The code, originally developed for Direct Numerical Simulations (DNS), uses fourth-order standard finite differences and a fourth-order low storage Runge-Kutta method by [13] for time integration.

The code uses characteristic interface conditions [14] for block communications with non-reflective zonal characteristic boundary conditions [15] to avoid discontinuities across mesh blocks. A sixth-order standard difference filter is also applied after every full Runga-Kutta time step to damp nu- 
merical instabilities. To further aid stability skew-symmetric splitting [16] of the convective terms is used.

The code employs the Wall Adaptive Local Eddy viscosity model by Nicoud and Ducros [17] to close the LES equations. The code was validated for supersonic wakes [18], turbulent jets in coflow [19], low pressure turbines [12], and axial compressors [9], providing evidence of its capabilities and performance. The turbulent inflow is achieved using the digital filter approach of Klein et al. [20], while the sliding mesh interface employs the same characteristic interface condition applied between block boundaries (see [21]).

The RANS simulations have been performed using the commercial code ANSYS CFX using the SST turbulence model with transition captured using the $\gamma-R e_{\theta}$ model. For the calculation of fluid viscosity Sutherland's law is used for both the LES and RANS simulations. The choice of the SST turbulence model for RANS modeling is made based on the transition model which is formulated in the context of the SST model.

The simulations were performed on the UK national super computer ARCHER using hybrid openmp-mpi parallelization. The time step was set to $1 \times 10^{-5}$ and $\approx 3 \times 10^{-5}$, normalised by chord and free-stream velocity, for the offdesign and wake cases respectively. the addition of wakes improved the stability of the simulation by changing the boundary layer behavior, allowing it to be run much closer to the CFL limit. The off-design simulation was run on 7,344 cores for 30 hours to obtain the statistical data needed while the wake case was run on 8,640 cores for 60 hours. Both cases required several flow through times to complete transition from initialization before statistics could be collected.

\section{RESULTS OVERVIEW}

This section focuses on validation, flow field analysis, and on the incidence sweep. The time averaging process was extended until no further change is seen with increased convergence time. The runs without incoming wakes required approximately 5 blade flow through times, while with wakes the through flow times extended to 10 . For the moving bar phase locked averages, a single bar period is broken down into 18 phases with each phase averaged over 10 instances.

In order to compare the various simulations from RANS and LES, an appropriate averaging technique is needed for the inlet and exit sections. Reference quantities were determined by using either mixed out averaging, as described by Prasad [22], or via mass averaging. For the clean inlet cases the total losses are calculated using mixed out averaging, while the total loss for the wake case is presented as a mass average loss to account for the unmixed wakes passing through the passage.

\subsection{Validation}

The reference experiments [10] and the LES concentrate on the V103 geometry at an inlet Mach number of 0.67 and a Reynolds number of 300,000. As described in the paper [9], the inlet flow angle must be adjusted from $\alpha=42^{\circ}$
Table 3. Comparison of the loss coefficient and turning angle between LES and experiment.

\begin{tabular}{|c|c|c|}
\hline & LES & Experimental [10] \\
\hline Loss coefficient & 0.045 & 0.042 \\
\hline Flow turning & $33.9^{\circ}$ & $34^{\circ}$ \\
\hline
\end{tabular}

to $40^{\circ}$ in the LES to match the experimental loading. The likely reason for this difference of $2^{\circ}$ is the presence of threedimensional effects in the experiments, which are discussed in Iseler, Hilgenfeld \& Pfitzner [23].

The LES loading is compared to the experimental loading in Figure 3. The peak isentropic Mach number is 0.90 in the LES compared to 0.87 in the data. The trailing edge isentropic Mach number is slightly lower in the LES than the experiment. These observations are consistent with the growth of the endwall boundary layers (i.e. a streamtube contraction) being present in the experiments. Such an acceleration from the leading edge to the trailing edge would explain why the experiment shows a lower isentropic Mach number than LES near peak suction but a higher isentropic Mach number towards the trailing edge.

The LES predicts a laminar separation and a separationinduced transition on the suction side around mid-chord. As is apparent from Figure 3, the separation occurs slightly earlier in the LES (between 35-55\% chord) compared with the experiment (between 40-60\%), which is consistent with the stronger adverse pressure gradient in the LES. The RANS however does not capture the suction side laminar separation and subsequent transition at all but instead predicts bypass transition. In addition to the loading, the losses and turning from LES and experiment also agree favourably, shown in Table 3.

For the case with upstream moving bars, Figure 4 compares the measured and predicted LES wakes. A velocity trace at a normal distance of $1.14 \%$ chord from the suction surface at an axial distance of $65 \%$ chord, Figure 5, and a momentum thickness trace at an axial position of $97 \%$ chord, Figure 6 confirm how the predictions are in excellent agreement with measurements.

For the momentum thickness, Figure 6, the comparison overall is good. However it must be noted that the normalization is done using the $44^{\circ}$ data from the "clean" inflow case, which is $1^{\circ}$ off. This may result in a slight scaling difference. The slight mismatch near the minimum momentum thickness is attributed to the presence of three dimensional effects in the experiment mentioned previously.

\subsection{Flow Field Analysis}

The instantaneous span-wise vorticity is shown in Figure 7 for the positive incidence case, wake case and low incidence case $\left(\alpha=37^{\circ}\right)$ from Leggett et al. [9]. The spanwise vorticity reveals the presence of large structures shedding from the pressure surface leading edge at low incidence, Figure 7(a), while they disappear at positive incidence, Figure 7(b). The suction surface shows the opposite trends with an attached boundary layer at negative incidence which transitions further down-stream while at positive incidence the 


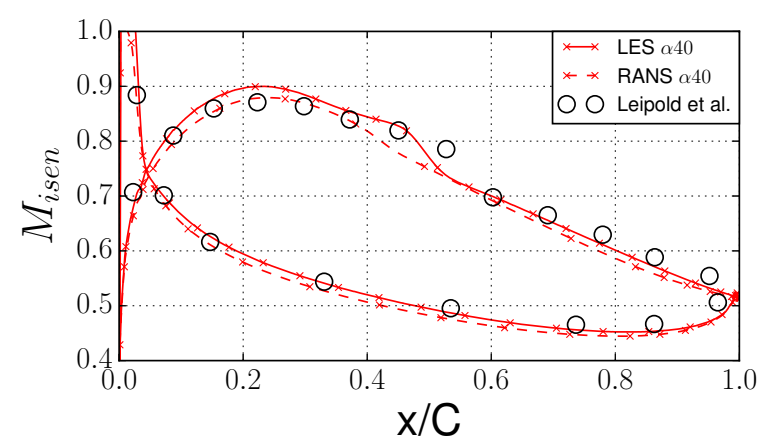

Fig. 3. Comparison of experimental loading from [10] and LES without moving bars at $40^{\circ}, \mathrm{Re}=300,000$, Mach 0.67

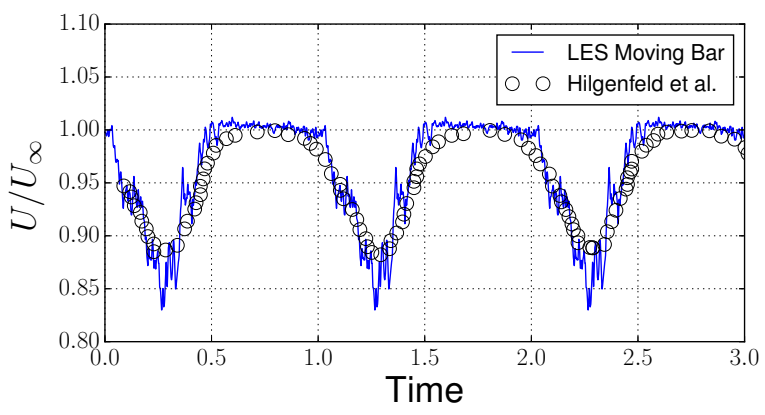

Fig. 4. Inlet velocity trace normalized with free stream velocity. Showing deficit produced by the moving bars.

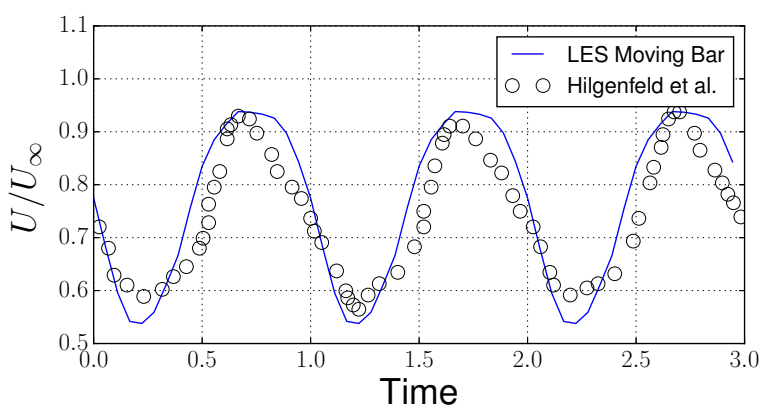

Fig. 5. Boundary layer Velocity trace at $1.14 \%$ chord normal from the suction surface at $65 \%$ axial chord for moving bar case.

suction surface shows a leading edge transition, though no large turbulent structures are present. For the wake case the span-wise vorticity, Figure 7(c), clearly shows the presence of the bar wakes passing through the blade passage, though the influence of the wakes on the boundary layer is slightly harder to see. It is still clear that both boundary layers are largely well behaved apart from where wake interaction occurs which induces transition.

The time averaged Turbulent Kinetic Energy (TKE) is given in Figure 8 for the same three cases illustrated above. The LES without wakes shows how the main source of turbulence in the flow moves from the pressure surface to the suction surface as incidence is increased. Interestingly, the main source of turbulence appears to be associated with the

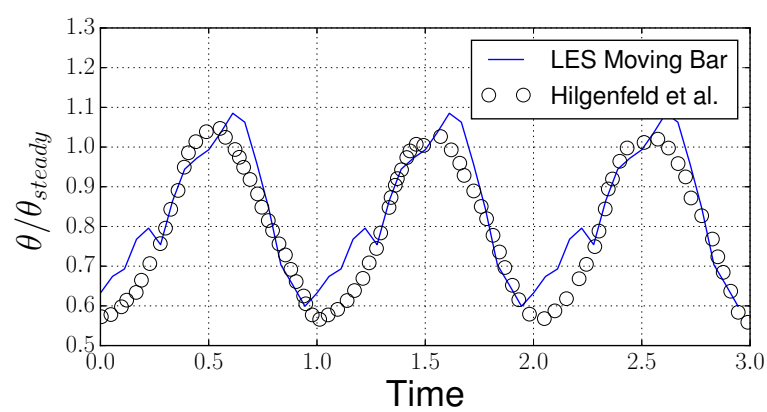

Fig. 6. Comparison of suction surface boundary layer momentum thickness at $97 \%$ axial chord for moving bar case. Normalised using the LES case at $44^{\circ}$.

trailing-edge separation on the suction surface at positive incidence, even though leading edge separation and transition is present in the high incidence case, as visible in the vorticity contours, Figure 7. For the wake case the TKE shows similar trends to the positive incidence off-design case, as the time average statistics shows the blade is running at a flow angle of $43^{\circ}$, which corresponds to a positive incidence angle.

\section{OFF-DESIGN CONDITIONS}

\subsection{Blade Loading and Skin Friction}

How the blade loading and the wall shear stress by RANS evolve from design to off-design are among the first things taken care of in the design phase. In this section the accuracy of RANS predictions are benchmarked with LES.

Figures 9,10 and 11 extend the blade loading and wall shear stress comparison by Leggett et al. [9] to a wider incidence range. At positive incidence there is a clear reduction in peak isentropic Mach number and no evidence of the suction side separation seen at negative incidence. RANS and LES predictions at positive incidence agree well, with only minor mismatch near the leading edge suction surface. The wall shear stress plot, Figure 11, shows how at positive incidence the pressure surface does not separate, with the boundary layer gradually transitionining to a turbulent state. The transition onset is slightly delayed on the pressure surface compared to the $44^{\circ}$ and the transition rate is also slower. RANS predicts the transition further downstream in comparison with the LES, without a clear increase in post transition skin friction. The slower transition predicted by RANS is in contrast to the isentropic Mach number loading, Figure 9, which shows a faster rate of deceleration of the pressure surface compared with LES. This suggests that the transition model in the RANS struggles to predict transition as well under these conditions. The slower transitions compared with the $44^{\circ}$ case is brought about by the leading edge stagnation point being located on the leading edge pressure side, reducing the flow acceleration around the leading edge on the pressure surface. This has the opposite effect on the suction surface, where a clear rapid acceleration/deceleration of the flow around the leading edge is seen in the sharp drop in the isentropic Mach loading plots. The remainder of the suction surface however has a similar steady deceleration from lead- 


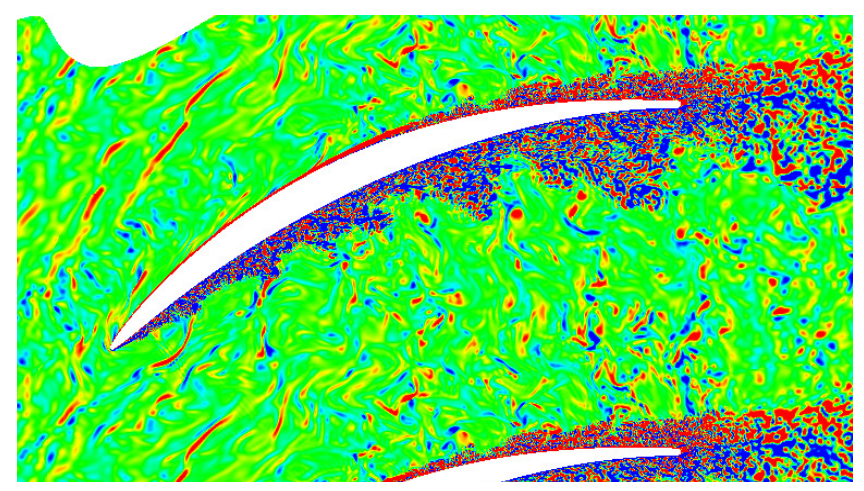

(a) $\alpha=37^{\circ}$

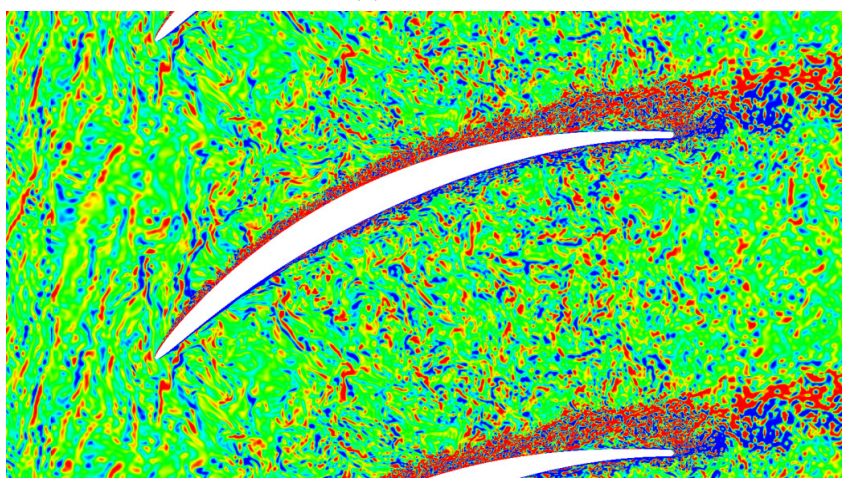

(b) $\alpha=49^{\circ}$

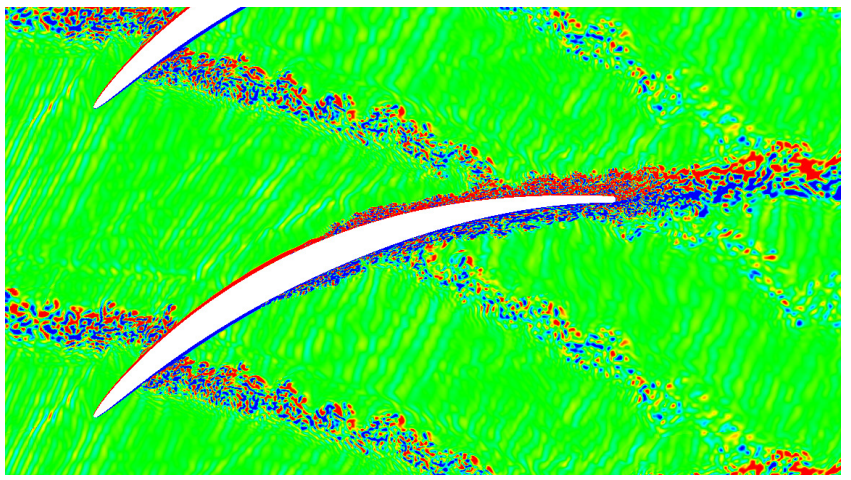

(c) Wake New Case

Fig. 7. Span-wise instantaneous vorticity for negative incidence case (a), positive incidence case (b) and wake case (c). Vorticity contour limits $[-10,10]$ based on non-dimensional velocity normalised by inlet reference.

ing to trailing edge and no longer shows acceleration to peak suction. However, as shown in Figure 10, the suction surface does undergo early transition near the leading edge. As the flow accelerates and decelerates around the leading edge curvature there is a short separation bubble that transitions immediately in the LES, while it shows a laminar reattachment and then transition in the RANS (see Figure 10). In this case, a fully-turbulent RANS, not shown here, predicts the boundary-layer behavior and wake profiles better than the RANS with transition as the fully turbulent results are closer to LES. This leading edge separation is a process that has been investigated by Walraevens and Cumpsty [24] who showed the effects of leading edge geometry and incidence at low speed. They also showed that Reynolds number has

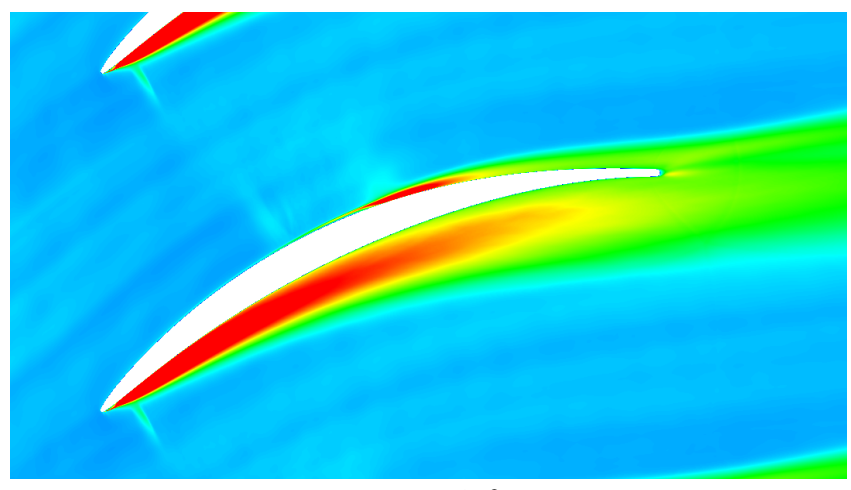

(a) $\alpha=37^{\circ}$

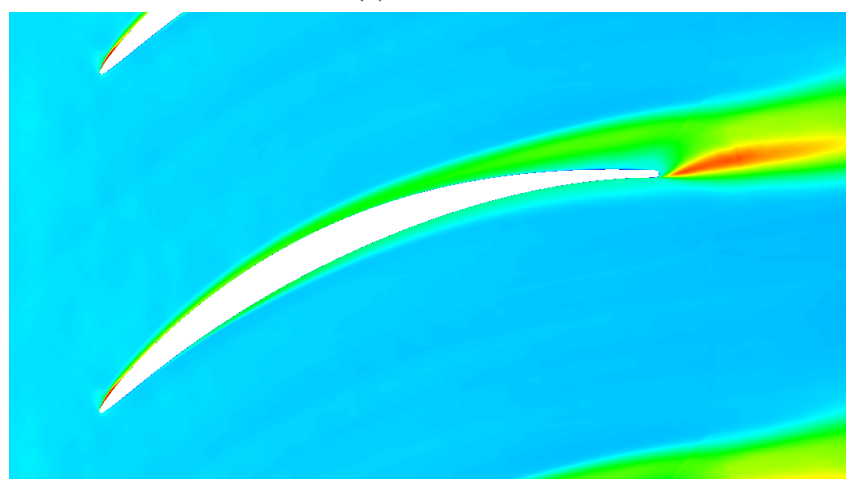

(b) $\alpha=49^{\circ}$

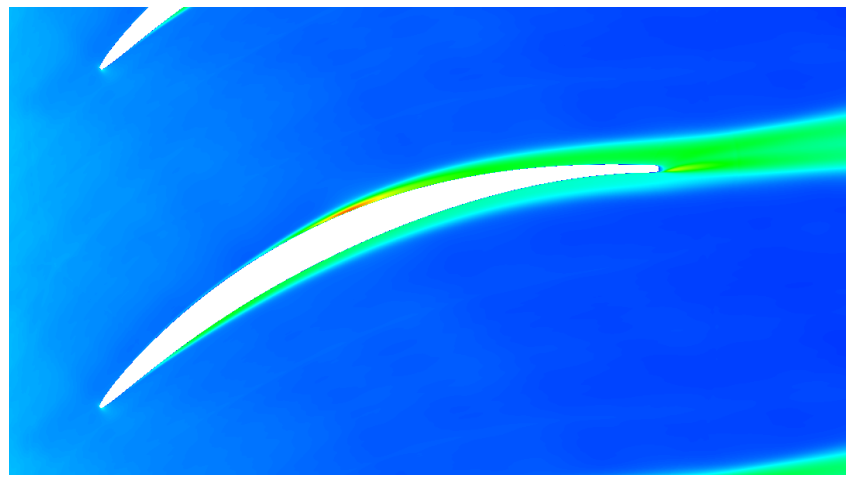

(c) Wake New Case

Fig. 8. TKE for off-design incidences $37^{\circ}$ (a), $49^{\circ}$ (b) and time statistics for the wake case (c). TKE contour limits $[0,0.2]$ normalised by inlet velocity.

limited effects on this leading edge separation. The rapid transition in the LES is likely due to the interaction of the free stream turbulence forcing the leading edge separation shear layer. It is also noted that the flow separates again at $\mathrm{x} / \mathrm{C} \approx 0.8$ and remains separated till the trailing edge, captured by both RANS and LES.

\subsection{Boundary Layer Profiles}

This section compares the LES and RANS boundary layer profiles to determine how these two methods predict the impact of the stream wise pressure gradient. Figures 12 and 13 show the suction surface wall normal profiles for TKE and tangential velocity respectively. The plots show the changes in the boundary layer from $\mathrm{x} / \mathrm{C}=0.64$ to 0.99 for the offdesign incidence cases. The changes in the wall normal pro- 


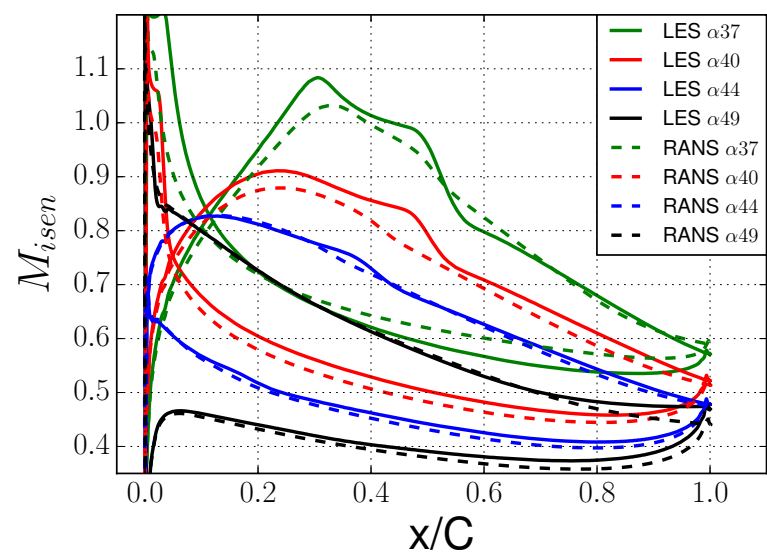

Fig. 9. Isentropic Mach number for off-design LES and RANS cases. RANS(dashed), LES(solid)

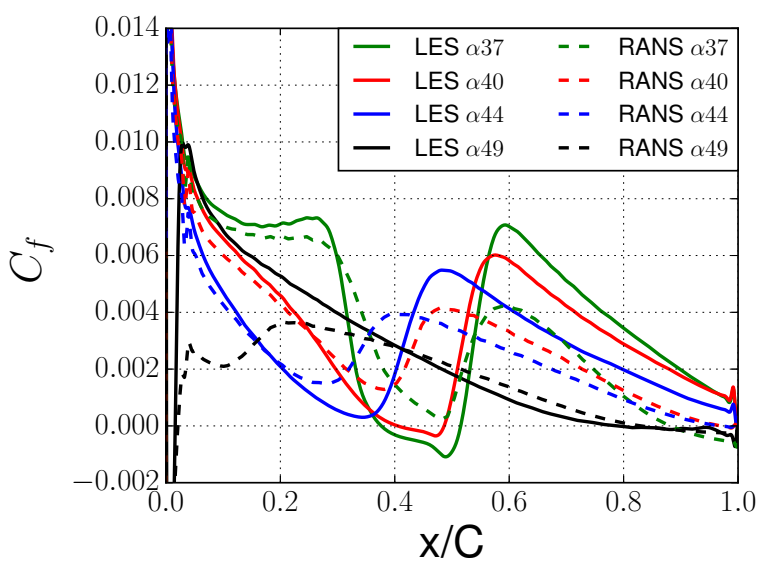

Fig. 10. Suction surface skin friction for off-design LES and RANS cases. RANS(dashed), LES(solid)

files support the changes seen in the skin friction and blade loading. The small differences between RANS and LES at the leading edge are propagated and amplified moving downstream, with a clear difference in velocity seen in the RANS profiles and less severe reversed flow at the trailing edge separation. The difference is also highlighted in the TKE where the LES shows a stronger free shear at the trailing edge and a higher level of TKE in the boundary layer, similar to the lower incidence cases.

\subsection{Wake Profiles}

As the interaction between adjacent blade rows is predominantly governed by wakes generated by the upstream row, Figures 14 and 15 compare the wake profiles by LES and RANS for two down stream planes at $2 \%$ and $10 \%$ chord from the trailing edge respectively.

In both figures, the LES profiles show vertical error bars that reflect the residual noise responsible for slightly negative losses in the flow core. As discussed in the Loss Breakdown section, this noise is quite small (the vertical bars are barely visible). When adding the residual noise value to the flow

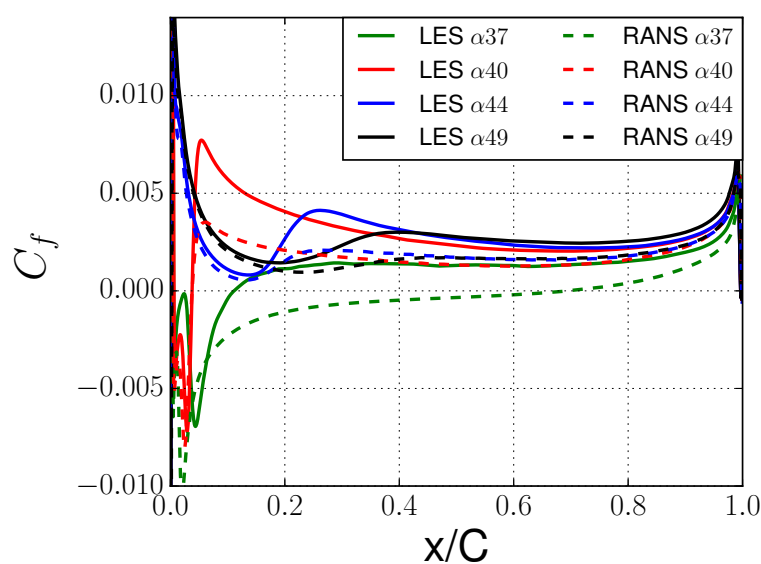

Fig. 11. Pressure surface skin friction for off-design LES and RANS cases. RANS(dashed), LES(solid)

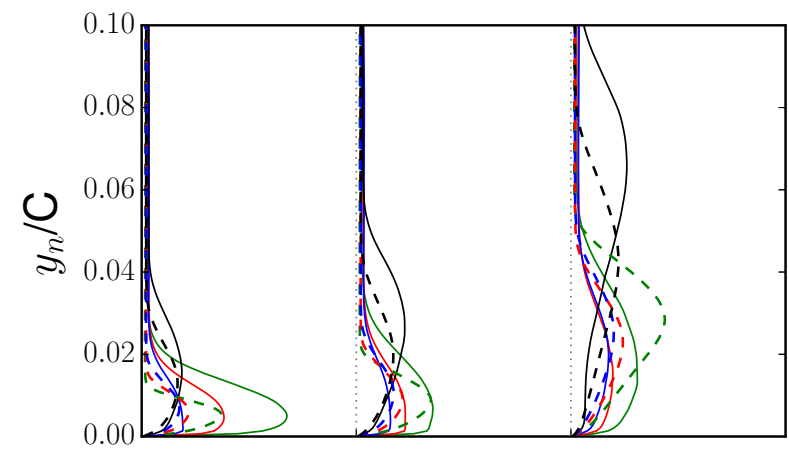

Fig. 12. Suction surface wall normal TKE profiles. At stream wise positions $x / C=0.64,0.76,0.99$, normalised with $U_{\infty}^{2}$. Profiles are offset by 0.05 along the $x$ axis. $\left[37^{\circ}\right.$ (green), $40^{\circ}$ (red), $44^{\circ}$ (blue), $49^{\circ}$ (black)]. RANS(dashed), LES(solid)

regions away from the wake, the losses turn positive again.

LES generally predicts wider wakes, with a more substantial deficit produced by the suction surface at positive incidence: a trend expected from the wall normal velocity profiles discussed previously. It is seen that the shear layer produced at negative incidence, produced by the mismatch in velocity of the pressure and suction surface boundary layers, is largely absent at positive incidence. A mismatch in velocity between the two blade surfaces is still present but as the pressure surface produces a very thin boundary layer the shear layer produced in the wake is minimal. The difference in the magnitude of the wake deficit between LES and RANS is comparable to that at negative incidence but reversed with the LES predicting a deeper wake at positive incidence while RANS predicts a deeper wake at negative incidence. Finally the predicted wake path for the LES shows the wake migrating toward the suction surface as it progresses down stream, a trend not shown by the RANS.

\subsection{Boundary Layer Quantities}

The trailing edge momentum thickness in Figures 16 and 17 for the pressure and suction surfaces respectively cor- 


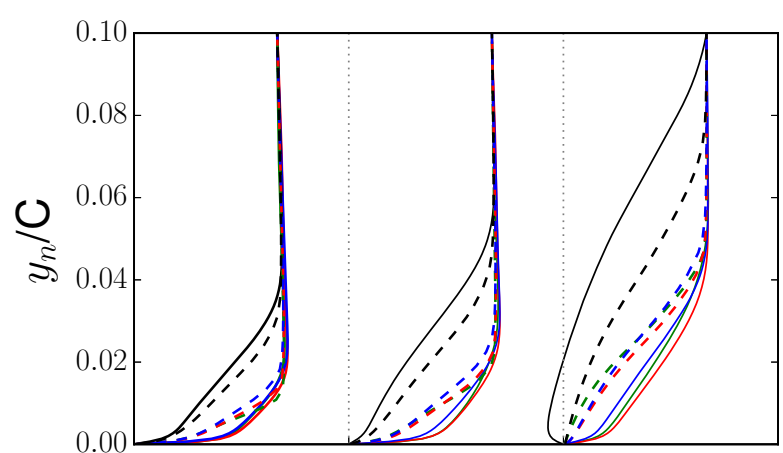

Fig. 13. Suction surface wall normal tangential velocity profiles at the same stream wise locations as Figure 12, normalised with the local free stream velocity. Profiles are off-set by 1.5 along the $x$ axis. [37 ${ }^{\circ}$ (green), $40^{\circ}$ (red), $44^{\circ}$ (blue), $49^{\circ}$ (black)]. RANS(dashed), LES(solid)

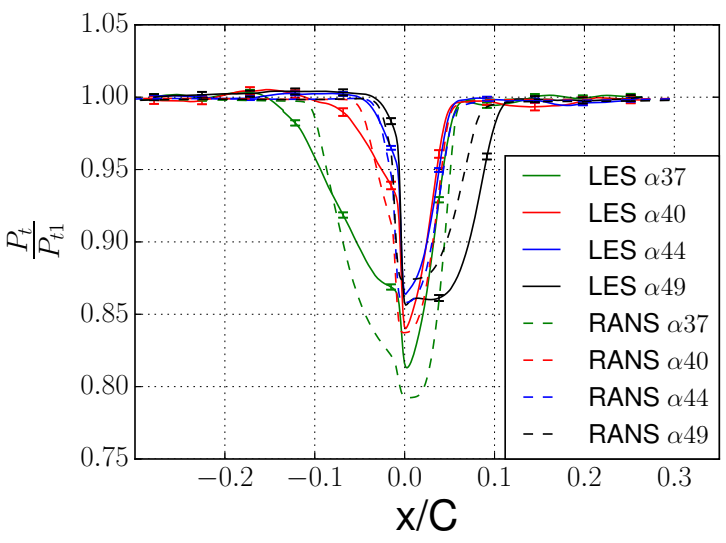

Fig. 14. Total pressure wake profile taken $2 \%$ chord downstream of the trailing edge.

relate to profile losses. It is therefore important to compare how LES and RANS predict this key parameter. It is seen that for both the pressure and suction surfaces there is some disagreement between RANS and LES at large negative or positive incidence. For the pressure surface this disagreement is worst at negative incidence though the agreement improves greatly as the incidence is increased. For the suction surface the opposite is true where disagreement is worst at positive incidence.

\section{LOSS BREAKDOWN}

Prior to proceeding with the losses breakdown, the total mixed out loss coefficient for variable incidence angles is shown in Figure 18. The LES "loss bucket" trend is fairly well matched by the RANS, and they both agree well with the measured value at $\alpha=40^{\circ}$. Leggett et al. [9] already discussed the difference in performance at low incidence, which will therefore only by lightly touched upon here when comparison is required. The LES predicted values are supplemented by vertical bars that represent the uncertainty level proportional to the residual noise at exit observed in the sim-

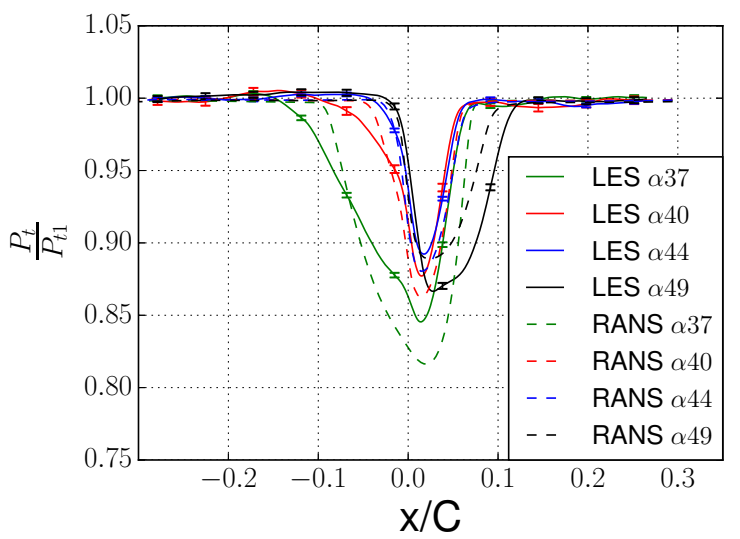

Fig. 15. Total pressure wake profile taken $10 \%$ chord downstream of the trailing edge.

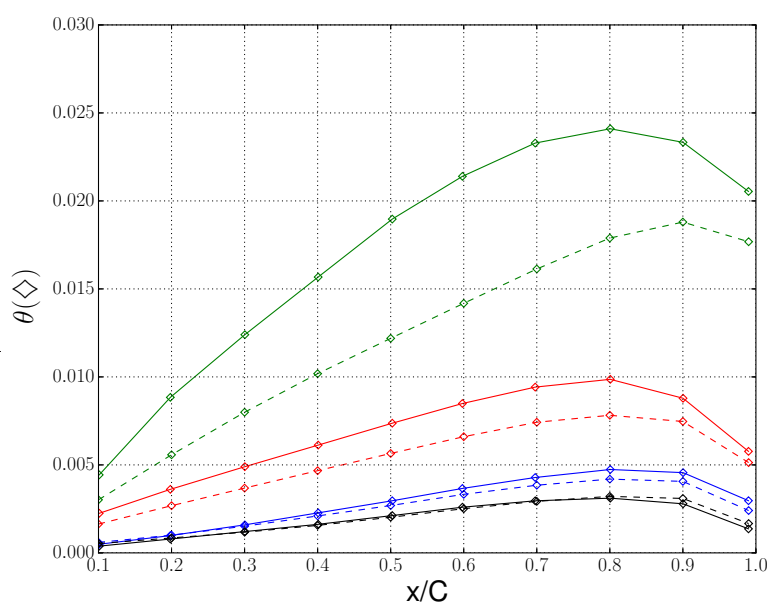

Fig. 16. Momentum thickness of the pressure surface for $37^{\circ}$ (Green), $40^{\circ}$ (Red), $44^{\circ}$ (Blue), $49^{\circ}$ (Black) with RANS(dashed) LES(solid).

ulations as discussed below. This uncertainty is very small, and it does not alter the overall LES predictions trend and the relative comparison with RANS. At positive incidence it is seen that RANS and LES match the loss well, improving on the comparison seen at $44^{\circ}$, where RANS predicts a notably higher loss. RANS and LES predictions are similar at $40^{\circ}$ and $49^{\circ}$ as the boundary layers at these off-design incidences are driven strongly by the dynamics of the flow, while at $44^{\circ}$ the boundary layer is driven by a more uniform pressure field which requires more accurate prediction by the models.

While the overall loss coefficient computed from the predicted time averaged exit stagnation pressure profile is in good agreement with measurements, the simulations revealed that without incoming wakes the exit stagnation pressure in the flow core away from the blade boundary layers could locally exceed the inlet value by less than $0.1 \%$. We never observed this phenomenon in the downstream wake, as well as in a quite large set of companion simulations performed on low-pressure and high-pressure turbines [6, 25]. After intense numerical testing, that included grid sensitiv- 


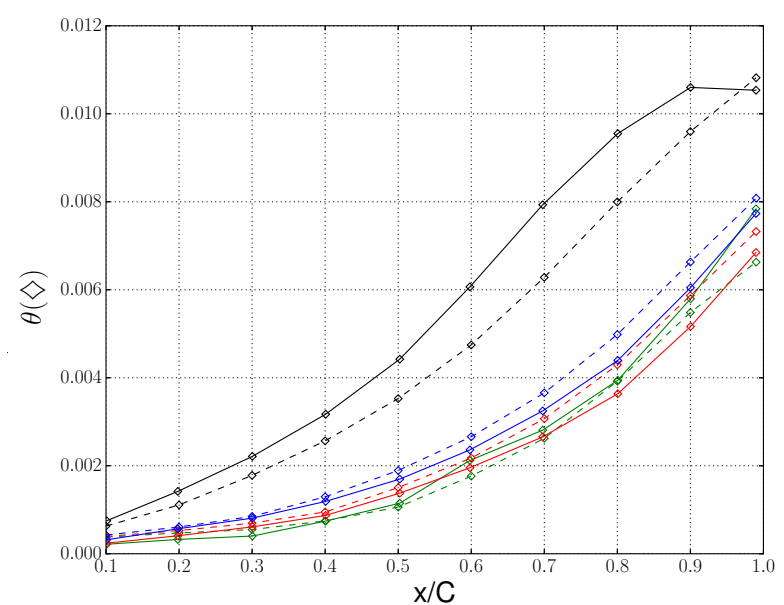

Fig. 17. Momentum thickness of the suction surface for $37^{\circ}$ (Green), $40^{\circ}$ (Red), $44^{\circ}$ (Blue), 49 ${ }^{\circ}$ (Black) with RANS(dashed) LES(solid).

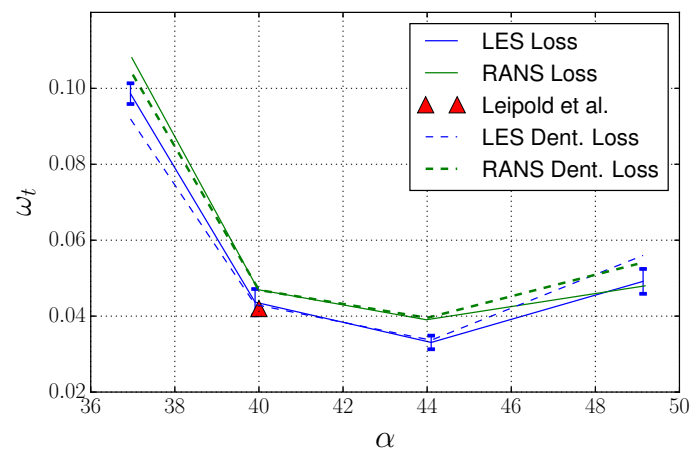

Fig. 18. Mixed out loss bucket for off-design incidence cases. The total losses are shown as solid lines while the total Denton loss breakdown is shown by dashed lines.

ity and statistical convergence analyses, we concluded this problem is caused by the concerted action of the extremely low-level of losses in the flow core and the inlet stagnation pressure profile dis-uniformities. The LES is conducted by specifying a flat time averaged inlet velocity and the inlet stagnation pressure is a result of the calculations. Due to the low inlet Mach number, potential effects distort the pitchwise inlet stagnation pressure profile by around $0.8 \%$ of the inlet average. Therefore, the average inlet value necessary to compute a loss coefficient may be smaller than local exit stagnation pressure values, also considering that losses in the flow core are negligible, and that the blockage in the blade passage causes a pitch-wise migration of the inlet minimum and maximum stagnation pressures. As soon as entropy production ramps up, i.e. in the wake, the exit stagnation pressure never exceeds the inlet value. To account for this residual noise in the calculations we added an error bar of approximately 5\% proportional to the local computed average negative loss, that does not alter the overall conclusions drawn from the simulations.

In order to provide useful information to designers an attempt is made to determine the origins of losses following
Denton [26] control volume analysis applied to the trailing edge of the compressor cascade. By performing a momentum, energy and mass balance between the trailing edge section and a far down stream section, the analysis allows the origins of blade loss to be associated with different boundary layer processes, measured at the trailing edge. The analysis by Denton results in the following expression

$$
\omega=-\frac{C_{p b} t}{w}+\frac{2 \theta}{w}+\left(\frac{\delta *+t}{w}\right)^{2},
$$

which describes the loss of a compressor cascade in terms of form or pressure loss, momentum loss and displacement or blockage loss respectively. Leggett et al. [9] applied the Denton loss breakdown analysis to the same NACA 65 cascade at negative incidence. As such breakdown proved quite sensitive to the definition of the boundary layer limit, the suggested method outlined by Leggett et al. will be used here in computing the boundary layer properties at $99 \%$ chord between two adjacent blades. Figure 18 compares the total loss calculated using the loss breakdown (dashed lines) with the total pressure loss coefficient. The agreement is generally good, although some difference arise at positive incidence. The Denton loss breakdown can be used to allocate loss to the various processes outlined separately for the pressure and suction surfaces, as shown in Figure 19 that reports only the dominant terms.

The main contribution to loss shifts from the pressure surface to the suction surface as the incidence is increased. Notably, the main contribution at low incidence comes from momentum loss from both surfaces. As expected at positive incidence the pressure surface contributes very little to losses, but the suction surface displacement loss becomes relevant. The higher contribution of displacement loss at positive incidences is more in-line with the loss breakdown trends seen for low pressure turbines outlined in the work by Michelassi et al. [4]. It is suggested that this increase in displacement loss is responsible for the over prediction in total loss calculated using the loss breakdown, as the loss prediction is very good at $44^{\circ}$ and dominated by momentum losses.

The comparison of the total Denton loss between RANS and LES is fair as both show the same trends with incidence. The main difference between RANS and LES is seen in the loss breakdown where there is reasonable difference in the relative contributions from each term. RANS predicts lower momentum and displacement losses compared with LES, with the discrepancy in the momentum loss largest at the limits of the loss bucket. Moreover, RANS does not predict the large change in suction surface displacement loss seen in the LES which is likely due to the change in the suction surface boundary layer discussed earlier where RANS shows a more energized boundary layer at the trailing edge. railing edge. The analysis by Denton resulted in the following expression 


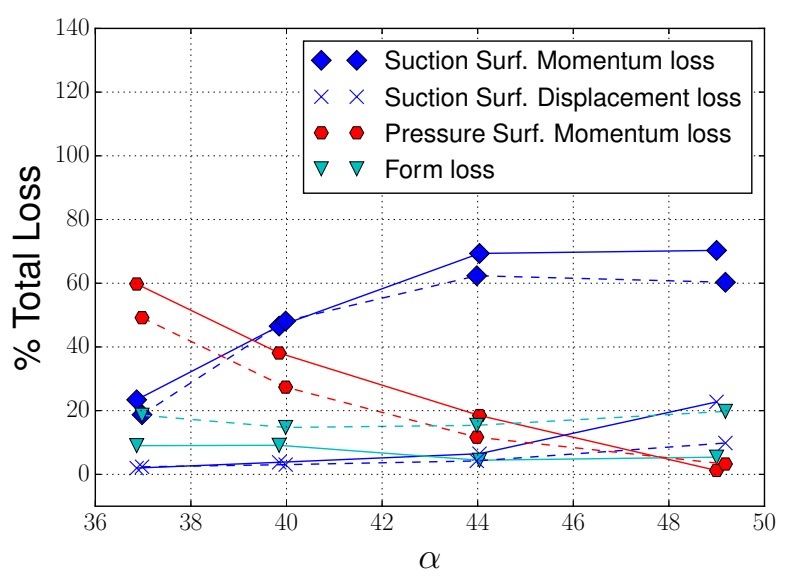

Fig. 19. Loss breakdown for off-design cases. Showing percentage of loss attributed to each term.
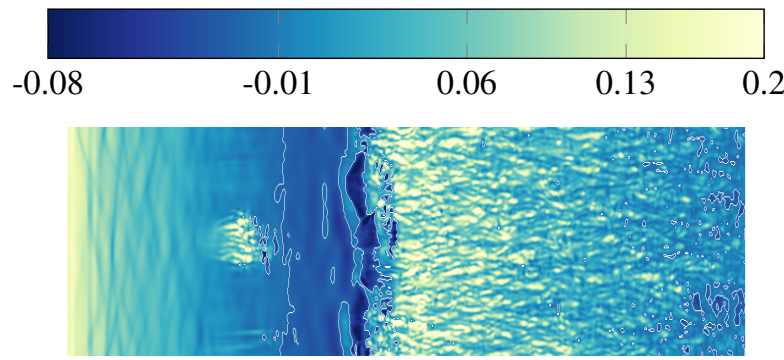

phase 8 (1)

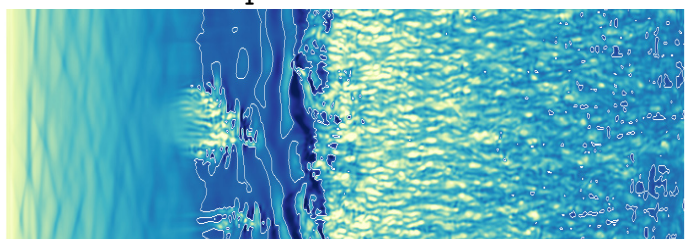

phase 10 (2)

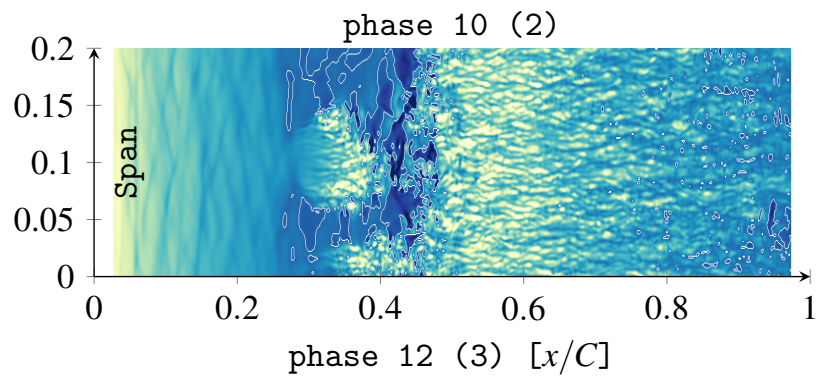

Fig. 20. Carpet plot showing the coalescing of wake induced turbulence and separation turbulence in the instantaneous tangential velocity. The plot is a plane $y_{n} / C=9 e-5$ from suction surface with zero velocity contour in white.

\section{INCIDENT WAKES}

The following section looks at the behavior of the blade boundary layer and loss when exposed to incident wakes generated by a set of upstream moving bars. The investigation with upstream moving bars by Hilgenfeld \& Pfitzner [11] used a non realistic combination of reduced frequency and flow coefficient $(0.66,5.7)$ with incoming wakes almost parallel to the compressor profile, due to the upstream bar

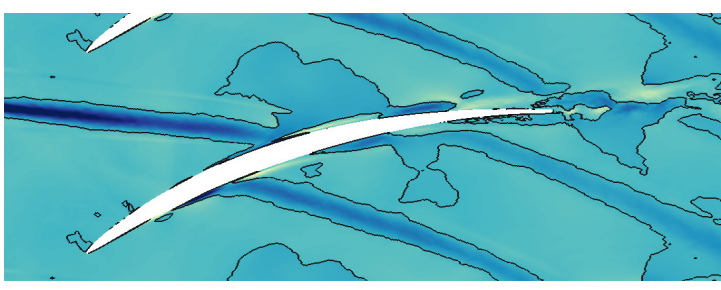

phase 8 (1)

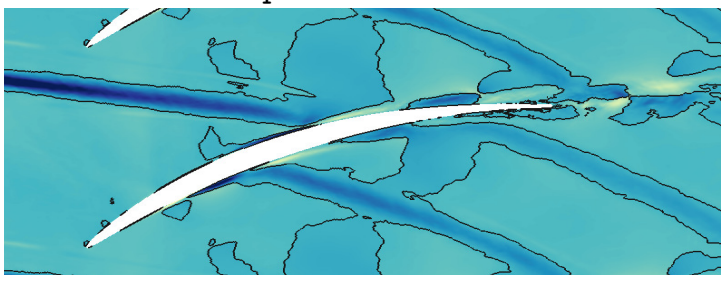

phase 10 (2)

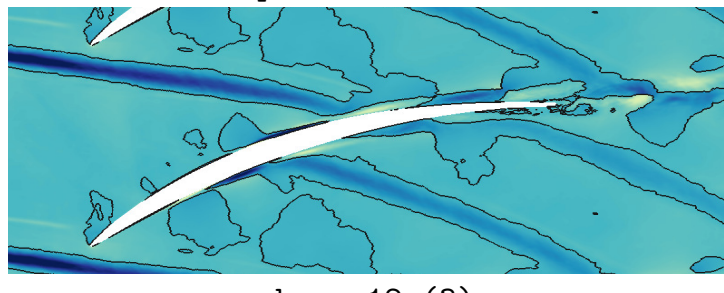

phase 12 (3)

Fig. 21. Contour plots of phase lock averages showing the velocity difference between the phase averages and full statistics, $\left\|U_{\text {phase }}\right\|-\left\|U_{\text {full }}\right\|$. Corresponding to the phases shown in Figure 20. The contours limits are $[-0.1,0.1]$ with zero limit shown in black.

tangential speed limitation. Therefore we designed a new numerical experiment by increasing the bar speed and reducing the bar count that gave a more realistic combination of $F_{\text {red }}$ and flow coefficient of $\approx 1.9$ and $\approx 0.89$ respectively, as summarized in Table 2. Due to page limitations the detailed analysis of this case will be discussed in a future paper but it is instructive to compare here some of the findings of the incoming wakes with the free-stream turbulence case of the same incidince.

\subsection{Incident Wake Flow Field}

The instantaneous time plots of Figure 20, in which the $x$-axis is the axial distance and the $y$-axis represents the $20 \%$ chord computational domain extension in the span-wise direction, show the tangential velocity at $y_{n} / C=9 e-5$ from the suction surface. The set of images in Figure 20 show the progression of wake induced turbulence and the consequent suppression of the separation bubble, seen in images (1) to (3). The zero velocity contour shown in white, outlines the areas of instantaneous separated flow. The position of the incoming wake on the suction side can be determined by looking at the companion plot of the velocity difference between the phase locked and full statistics of Figure 21.

Looking at the instantaneous data of the various simulations provides a good means of understanding what processes are involved. In particular, the three snapshots reveal the presence of Kelvin-Helmoltz $(K-H)$ instabilities with 


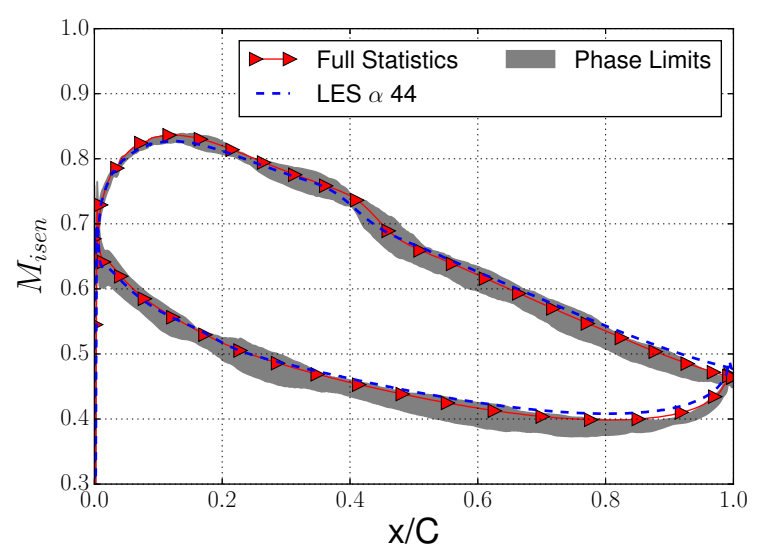

Fig. 22. Isentropic Mach number for incident wake case and offdesign case. Phase lock average extremes shown in gray.

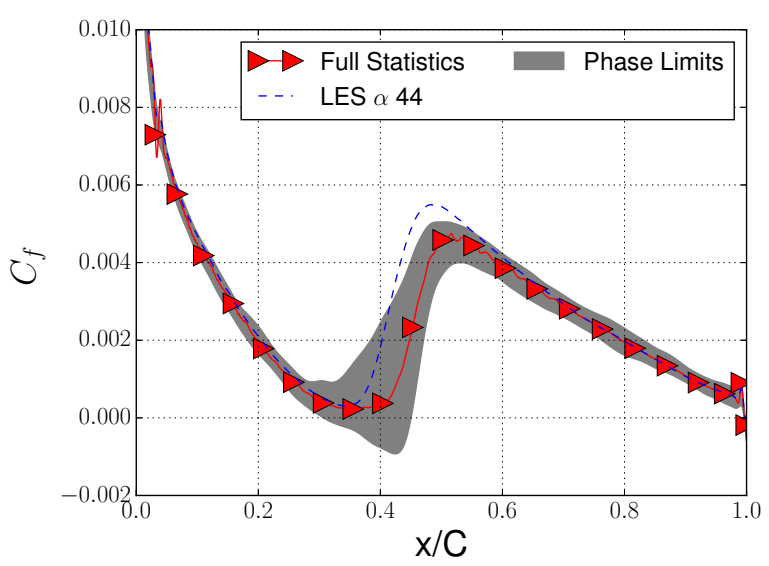

Fig. 23. Suction surface skin friction for off-design and incident wake cases. Phase lock average extremes shown in gray.

the intermittent presence of small separations that are the premise to the subsequent transition to a turbulent state of the boundary layer. It appears that the $K-H$ structures are very moderately triggered by the incoming wakes. The incoming wakes are responsible for the local growth of turbulent kinetic energy visible as a quasi-circular spot at around 0.25 span moving 0.3 to 0.35 in the streamwise direction. The size and intensity of the spot grows until it merges with more turbulent zone for $x / c>0.45$.

Figure 21 shows the so-called velocity difference between the phase locked and full statistics, thereby allowing a clear identification of the incoming wake path. The wakes remain coherent and narrow, and they look very moderatly affected by the local pressure gradient induced by the presence of vanes. The velocity deficit does reach the vane suction side in the front part of the profile, while further downstream the suction side boundary layer growth seems to shield the profile from the direct impact of the wake. This is also confirmed by the quasi-steady nature of the flow turbulence intensity for $x / C>0.5$ in Figure 20 .

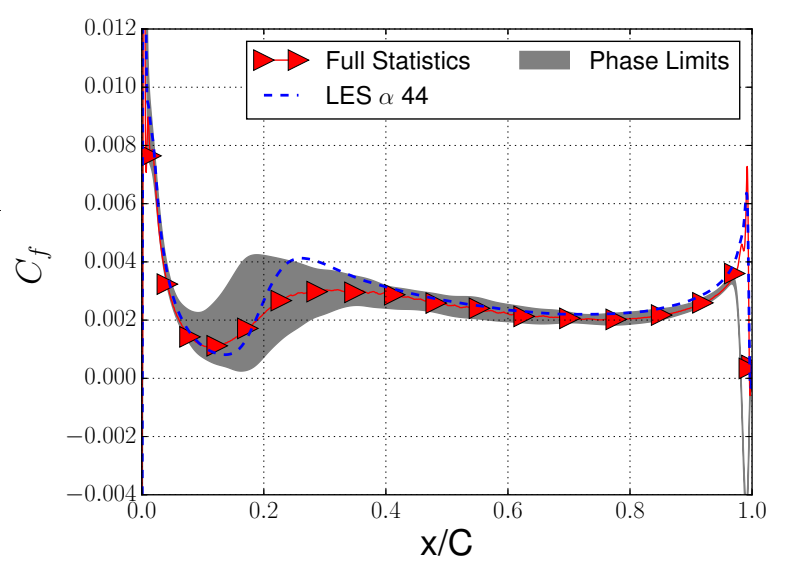

Fig. 24. Pressure surface skin friction for off-design and incident wake cases. Phase lock average extremes shown in gray.

\subsection{Unsteady blade loading and skin friction}

The blade loading and skin friction are shown in Figures 22, 23 and 24, for the full time-averaged statistics and the limits of the phases. For comparison, the figures also incorporate the companion $\alpha=44^{\circ}$ case without incoming wakes. The steady and time-averaged unsteady load distributions are very similar, and the unsteady load fluctuations are very limited all along the profile. Still, the trailing edge steady $M_{i s e n}$ is slightly above the time-averaged unsteady value, thereby suggesting that the incoming wakes may have a beneficial effect on the pressure recovery. The suction side skin friction plot of Figure 23 shows that the periodic incoming wakes may provoke a local separation that re-attaches soon, and never later than $x / C=0.45$. While the steady skin friction shows an earlier transition, the steady and time-averaged unsteady remarkably collapse for $x / C>0.55$. Probably, the absence of a suction side separation in the steady flow predictions can be imputed to this early transition. With the exception of the presence of an intermittent separation, the steady and the unsteady results remain quite similar when approaching the trailing edge. The pressure side skin friction plots of Figure 24 show a trend that closely resembles that of the suction side. Here the steady and unsteady predictions are again quite similar, although the presence of the incoming wakes seems to smear the position of transition of the boundary layer. Once again, while the unsteady predictions slightly deviate from the steady, they do collapse in the aft portion of the profile for $x / C>0.4$. The suction and pressure side plots reveal very moderate differences between the steady and unsteady predictions in the aft portion of the profile, which is in agreement with what the carpet and isentropic Mach number plots show.

\section{CONCLUSIONS}

The computations revealed that at design conditions both RANS and LES predict a loss level in good agreement with measurements. When moving off design, and using LES as a benchmark, RANS showed the correct trend with departures from the loss levels predicted by LES below 
0.005, as visible in Figure 18. The same fair agreement between RANS and LES is confirmed for the isentropic Mach number distribution around the airfoil for the four incidence conditions investigated here (see Figure 9). While this agreement may be satisfactory for a preliminary design investigation, more fundamental differences arose when deepening the comparison. In particular:

- The comparison of the momentum and displacement thickness, that indicate loss levels and blockage respectively, on both the pressure and suction sides, reveals that RANS does a fair job around the design point, while more substantial differences, attributable to the turbulence model, grow at off-design (for momentum thickness see Figures 16, 17).

- The nature of the loss generation predicted by LES and RANS shows some important differences. From the loss breakdown it is seen that the main sources of loss change as incidence is varied. LES and RANS show a different growth of momentum, displacement, and form losses, culminating at $\alpha=49^{\circ}$. In particular, the relative importance of suction and pressure side losses are different between RANS and LES. This alone can have an important impact in guiding the process to improve the design of the airfoil.

- At positive incidence the pressure surface plays very little part in loss generation but the suction surface now contributes a reasonable displacement or blockage loss as well as a high momentum loss.

- The predicted wakes, both in the immediate proximity of the trailing edge, and further downstream, reveal substantial differences between LES and RANS at off design conditions. For the negative incidence case, the shape of the wakes is different, although they seem to carry a similar level of losses. This implies that LES will predict a different interaction with downstream blade rows as compared to RANS. Similar considerations hold for the positive incidence case, while closer to design the wakes are in good agreement.

- The results show differences in the boundary layer separation and transition behavior between LES and RANS. At positive incidence $\left(\alpha=49^{\circ}\right)$, LES predicts a leading edge separation with turbulent reattachment on the suction side. RANS (with the transition model) captures the leading edge separation but the reattachment is predicted to be laminar, rather than turbulent, with transition occurring further downstream through a bypass mechanism. A fully-turbulent RANS, not shown here, gives a better prediction of the boundary layer behavior and wake profiles in this case.

- The unsteady case with periodic moving bars revealed on first inspection very limited variation in loading with some changes in the suction side skin friction where the periodic wakes delay the transition point resulting in the growth of an intermittent separation.

In general it is seen that RANS underestimates the relative displacement and momentum losses, at both positive and negative incidence, but predicts a larger pressure loss, which remains almost constant over the incidence sweep. We link the loss trends as a function of incidence, as well as the loss differences between RANS and LES, to the physical details of the predicted flow fields. Differences in the boundarylayer behavior, including the separation and transition behavior, are highlighted.

The simulations show that RANS is indeed a valid alternative to LES as long as the designer concentrates on profile pressure distributions and overall loss trends. Conversely, corrective actions aimed at reducing losses and blade-row interaction by controlling the shape of the wakes, may be biased by the inability of RANS to predict the correct loss split and off-design wakes shapes.

\section{Acknowledgements}

Acknowledgment is given to the UK turbulence consortium for the provision of computational time on the UK national computer Archer under grant number EP/L000261/1. The first author would like to acknowledge the financial support from the EPSRC and General Electric.

\section{Nomenclature}

$\begin{array}{ll}\mathrm{w} & \text { Airfoil pitch } \\ C & \text { Axial chord } \\ \mathrm{x} & \text { Axial distance } \\ P_{b a r} & \text { Bar pitch } \\ V_{b a r} & \text { Bar velocity } \\ C_{p b} & \text { Base pressure coefficient } \\ \delta^{*} & \text { Displacement thickness non-dimensionalized } C \\ \alpha & \text { Inlet flow angle } \\ M_{i s e n} & \text { Isentropic Mach number } \\ \theta & \text { Momentum thickness non-dimensionalized } C \\ \mathrm{y} & \text { Pitch distance } \\ \omega & \text { Pressure loss coefficient }\left(\frac{P_{t 1}-P_{t 2}}{P_{t 1}-P_{1}}\right) \\ F_{\text {red }} & \text { Reduced frequency } \\ \mathrm{P} & \text { Static pressure } \\ \mathrm{P}_{t} & \text { Total pressure } \\ \text { Tu } & \text { Turbulence intensity }\left(\frac{\sqrt{(T K E)}}{U_{1}}\right) \\ U & \text { Velocity } \\ \text { Abbreviation } \\ \text { CFD } & \text { Computational fluid dynamics } \\ \text { DNS } & \text { Direct numerical simulation } \\ \text { LES } & \text { Large eddy simulation } \\ \text { RANS } & \text { Reynolds averaged Navier Stokes } \\ \text { TKE } & \text { Turbulent kinetic energy } \\ \text { Subscripts } \\ 1,2 & \text { inlet, outlet plane } \\ \mathrm{n} & \text { wall normal } \\ & \end{array}$

\section{References}

[1] Adamczyk, J. J., Hansen, J. L., and Prahst, P. S., 2007. "A Post Test Analysis of a High-Speed Two-Stage Axial Flow Compressor”. In ASME Turbo Expo: Power 
for Land, Sea, and Air, Vol. 6 Parts A, ASME, pp. 377388.

[2] Lübcke, H., Schmidt, S. T., Rung, T., and Thiele, F., 2001. "Comparison of LES and RANS in bluff-body flows". J. Wind Engineering and Industrial Aerodynamics, 89(14-15), pp. 1471-1485.

[3] Yao, Y. F., Savill, A. M., Sandham, N. D., and Dawes, W. N., 2002. "Simulation and modelling of turbulent trailing-edge flow". Flow, Turbulence and Combustion, 68(4), pp. 313-333.

[4] Michelassi, V., Chen, L.-W., Pichler, R., and Sandberg, R. D., 2015. "Compressible Direct Numerical Simulation of Low-Pressure TurbinesPart II: Effect of Inflow Disturbances". ASME J. Turbomach., 137(7), jul, pp. 71005-71012.

[5] Pichler, R., Sandberg, R. D., Michelassi, V., and Bhaskaran, R., 2016. "Investigation of the Accuracy of RANS Models to Predict the Flow Through a LowPressure Turbine". ASME J. Turbomach., 138(12), jun, pp. 121009-121012.

[6] Michelassi, V., Chen, L., Pichler, R., Sandberg, R., and Bhaskaran, R., 2016. "High-Fidelity Simulations of Low-Pressure Turbines: Effect of Flow Coefficient and Reduced Frequency on Losses". ASME J. Turbomach., 138(11), may, pp. 111006-111012.

[7] Cumpsty, N. A., Dong, Y., and Li, Y. S., 1995. "Compressor Blade Boundary Layers in the Presence of Wakes". In ASME Turbo Expo: Power for Land, Sea, and Air, Vol. 1 Turbomac, ASME, p. V001T01A108.

[8] Adamczyk, J. J., Celestina, M. L., and Chen, J. P., 1996. "Wake-Induced Unsteady Flows: Their Impact on Rotor Performance and Wake Rectification". ASME J. Turbomach., 118, p. 88.

[9] Leggett, J., Sandberg, R., Michelassi, V., Priebe, S., and Shabbir, A., 2016. "Detailed Investigation of Rans and Les Predictions of Loss Generation". In ASME Turbo Expo: Power for Land, Sea, and Air, Vol. Volume 2A:

[10] Leipold, R., Boese, M., and Fottner, L., 2000. "The Influence of technical surface roughness caused by precision forging on the flow around a highly loaded compressor cascade". ASME J. Turbomach., 122, pp. 416425.

[11] Hilgenfeld, L., and Pfitzner, M., 2004. "Unsteady Boundary Layer Development Due to Wake Passing Effects on a Highly Loaded Linear Compressor Cascade". ASME J. Turbomach., 126(4), p. 493.

[12] Sandberg, R. D., Michelassi, V., Pichler, R., Chen, L., and Johnstone, R., 2015. "Compressible Direct Numerical Simulation of Low-Pressure TurbinesPart I: Methodology". ASME J. Turbomach., 137(5), p. 051011.

[13] Kennedy, C. A., Carpenter, M. H., and Lewis, R. M., 2000. "Low-storage, explicit RungeKutta schemes for the compressible NavierStokes equations". Applied Numerical Mathematics, 35(3), pp. 177-219.

[14] Kim, J. W., and Lee, D. J., 2003. "Characteristic Interface Conditions for Multiblock High-Order Computation on Singular Structured Grid". AIAA, 41(12).
[15] Sandberg, R. D., and Sandham, N. D., 2006. "Nonreflecting zonal characteristic boundary condition for direct numerical simulation of aerodynamic sound". AIAA, 44(2), pp. 3-6.

[16] Kennedy, C., and Gruber, A., 2008. "Reduced aliasing formulations of the convective terms within the NavierStokes equations for a compressible fluid.”. J. Сотp. Physics, 227, pp. 1676-1700.

[17] Nicoud, F., and Ducros, F., 1999. "Subgrid-Scale Stress Modelling Based on the Square of the Velocity Gradient Tensor". Flow, Turbulence and Combustion, 62(3), pp. 183-200.

[18] Sandberg, R. D., 2012. "Numerical investigation of turbulent supersonic axisymmetric wakes". J. Fluid Mech., 702, pp. 488-520.

[19] Sandberg, R. D., Sandham, N. D., and Suponitsky, V., 2012. "DNS of compressible pipe flow exiting into a coflow". Int. J. Heat and Fluid Flow, 35, pp. 33-44.

[20] Klein, M., Sadiki, a., and Janicka, J., 2003. "A digital filter based generation of inflow data for spatially developing direct numerical or large eddy simulations". J. Comp. Physics, 186(2), pp. 652-665.

[21] Johnstone, R., Chen, L., and Sandberg, R. D., 2015. “A sliding characteristic interface condition for direct numerical simulations". Comp. and Fluids, 107, pp. 165177.

[22] Prasad, A., 2005. "Calculation of the Mixed-Out State in Turbomachine Flows". ASME J. Turbomach., 127(3), p. 564.

[23] Iseler, J., Hilgenfeld, L., and Pfitzner, M., 2006. "Investigations of the Boundary Layer on a Highly Loaded Compressor Cascade With Wake-Induced Transition". In ASME Turbo Expo: Power for Land, Sea, and Air, Vol. 6 Part A a, ASME, pp. 1811-1820.

[24] Walraevens, R. E., and Cumpsty, N. A., 1995. "Leading Edge Separation Bubbles on Turbomachine Blades". ASME J. Turbomach., 117(1), pp. 115-125.

[25] Wheeler, A. P. S., Sandberg, R. D., Sandham, N. D., Pichler, R., Michelassi, V., and Laskowski, G., 2016. "Direct Numerical Simulations of a High-Pressure Turbine Vane". ASME J. Turbomach., 138(7), feb, pp. 71003-71009.

[26] Denton, J. D., 1993. "The 1993 IGTI Scholar Lecture: Loss Mechanisms in Turbomachines". ASME J. Turbomach., 115(4), p. 621. 